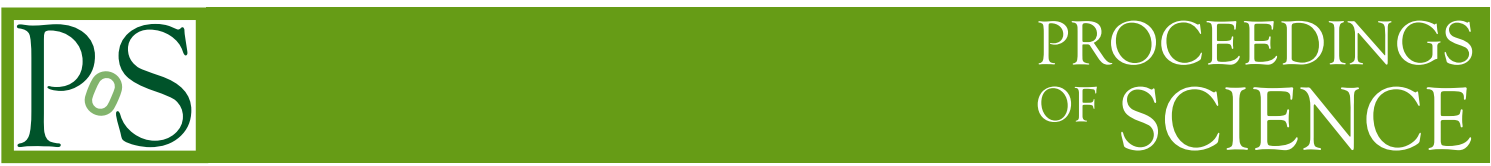

\title{
Enhanced CP violation in the Magnetic Field
}

\author{
Peter Filip* \\ Institute of Physics, Slovak Academy of Sciences, Dúbravská cesta 9, Bratislava 82103, Slovakia \\ E-mail: peter.filip@savba.sk
}

\begin{abstract}
We speculate about possible enhancement of $\mathrm{CP}$ - violating decays $\eta(547) \rightarrow \pi^{+} \pi^{-}$in a static, sufficiently strong magnetic field. The phenomenon is related to the superposition of quantum states with different $\mathrm{C}$ parity, occurring in $(J=0)$ ground state of pseudoscalar $\eta$ meson in the magnetic field. Existence of $\mathrm{G}$ parity violation in hadronic decays seems to be necessary for this phenomenon to occur. We also suggest CP - violating phenomena in the early (rotating) Universe could be enhanced by the presence of cosmological electroweak fields.
\end{abstract}

The European Physical Society Conference on High Energy Physics 22-29 July 2015

Vienna, Austria

${ }^{*}$ Speaker. 


\section{Introduction}

Electroweak sector of Standard Model [1] allows for the efficient description of CP violating phenomena in meson decays, using a single (CP phase) parameter in Cabibbo-Kobayashi-Maskawa (CKM) matrix [1]. Violation of CP symmetry due to mixing [1], which occurs in neutral meson decays, is related to the internal quantum state (superposition) of decaying pseudoscalar meson. Non-conservation of strangeness by weak interactions allows the initial $K^{0}=(d \bar{s})$ flavor state to evolve into $\bar{K}^{0}=(\bar{d} s)$ state, and the superposition of these flavor eigenstates becomes the ground state of neutral kaons in vacuum. Usually, one presumes, that parameters of this superposition (in the ground state of decaying meson) are not influenced by external fields.

In this contribution, we discuss a possibility of modifying the ground-state wavefunction of neutral mesons in such specific way, that decay branching ratio into CP eigenstate $\left(\pi^{+} \pi^{-}\right)$gets influenced. This allows us to speculate that $\mathrm{CP}$ violation could be influenced by the external fields.

\section{Starting from $K_{S}^{0}, K_{L}^{0}$ and comparing to $\mathbf{G}$ parity}

It has been observed experimentally, that neutral Kaons in vacuum exist in two different states $K_{S}^{0}$ and $K_{L}^{0}$, each having different lifetimes and decay properties [1]. Short-living state $K_{s}^{0}$, with lifetime $\tau=2.68 \mathrm{~cm} / \mathrm{c}$, was found to decay via $K_{s}^{0} \rightarrow \pi \pi$ channel in $99,89 \pm 0.1 \%$ of cases [1], while long-lived neutral Kaons (with 570x longer lifetime $\tau=15.3 \mathrm{~m} / \mathrm{c}$ ) are observed to decay via $K_{L}^{0} \rightarrow \pi \pi \pi$ channel (in $32 \%$ of cases) and $K_{L}^{0} \rightarrow \pi^{ \pm} l^{\mp} v_{l}$ (in $67,6 \%$ of cases). Mesons $K_{L}^{0}$ and $K_{S}^{0}$ were originally considered to be pure eigenstates $K_{1,2}^{0}=(d \bar{s} \pm \bar{d} s) / \sqrt{2}$ of weak interaction Hamiltonian, which was assumed to conserve CP symmetry exactly.
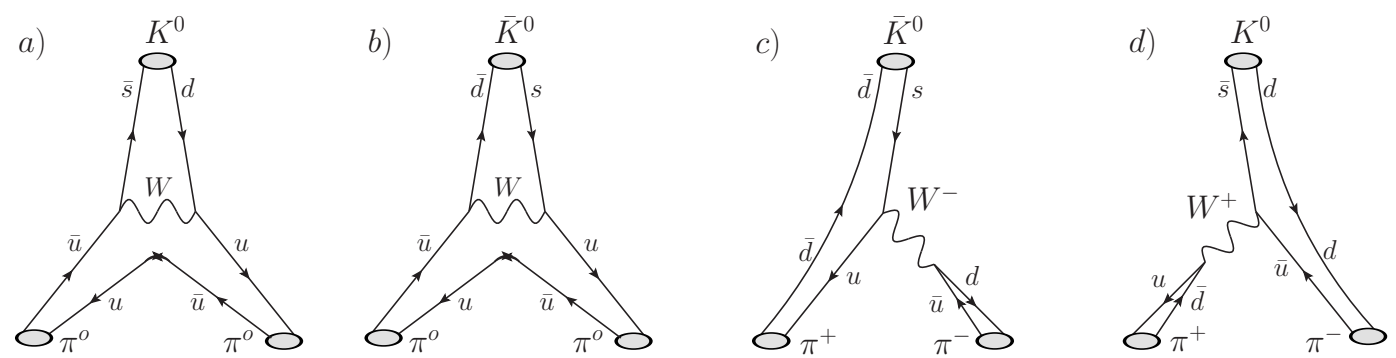

Figure 1: Example of weak decays of pure flavor states $(\bar{s} d)$ and $(\overline{d s})$ into two pions. For CP eigenstate $K_{2}^{0}=(\bar{s} d-\bar{d} s) / \sqrt{2}$, the interference of amplitudes $\left|A^{\bar{s} d}-A^{\bar{d} s}\right|^{2}$ gives zero probability for $K_{2}^{0} \rightarrow \pi \pi$ decays, provided that direct CP violation [3] in decay of $(\bar{s} d),(\bar{d} s)$ flavor eigenstates is zero (a slight simplification).

A surprise came with the observation [2] of $K_{L}^{0} \rightarrow \pi^{+} \pi^{-}$decays, many meters far from $(B e)$ internal target at AGS accelerator [2]. Authors of the pioneering paper [2] suggested $|\varepsilon| \approx 2 \cdot 10^{-3}$ admixture of CP eigenstate $K_{1}^{0}=(\bar{s} d+\bar{d} s) / \sqrt{2}$ would be present in the wavefunction of long-living mesons $K_{L}^{0} \approx\left(K_{2}^{0}+\varepsilon K_{1}^{0}\right)$, and the exciting CP violation story in particle physics was born.

\subsection{G parity violation in $\omega(782) \rightarrow \pi^{+} \pi^{-}$decays}

Let us consider now the violation of $\mathrm{G}$ parity in $\omega(782) \rightarrow \pi^{+} \pi^{-}$decays. In strong decays of hadrons, production of pure $(u \bar{u})$ and $(d \bar{d})$ flavor states occurs [4] via $(q \bar{q})$ QPC creation mechanism. Transitions $A_{\bar{d} d}^{u \bar{u}}=\left(u \bar{u} \rightarrow\left\{3 g, \gamma^{*}\right\} \rightarrow \bar{d} d\right)$ and $A_{\bar{u} u}^{d \bar{d}}=\left(d \bar{d} \rightarrow\left\{3 g, \gamma^{*}\right\} \rightarrow \bar{u} u\right)$ are allowed, and 
therefore, superpositions $\tilde{\rho}_{770}=(u \bar{u}-d \bar{d}) / \sqrt{2}$ and $\tilde{\omega}_{782}=(u \bar{u}+d \bar{d}) / \sqrt{2}$ become eigenstates [5] of the Hamiltonian of light $(q \bar{q})$ pairs. If masses and charges of $u, d$ quarks were identical, only strong decays $\tilde{\rho}_{770} \rightarrow \pi^{+} \pi^{-}$and $\tilde{\omega}_{782} \rightarrow \pi^{+} \pi^{-} \pi^{0}$ would be possible, with $G$ parity conserved.

However, electric charges and masses of $u$ and $d$ quarks are different [1], and therefore, small admixture $\varepsilon \cdot \tilde{\rho}_{770}$ is generated [6] in $\omega(782)=\left(\tilde{\omega}_{782}+\tilde{\varepsilon} \cdot \tilde{\rho}_{770}\right) / \sqrt{1+|\tilde{\varepsilon}|^{2}}$ meson state, which allows for (G parity violating) decays $\omega(782) \rightarrow \pi^{+} \pi^{-}$to occur [1].

In this scenario, $G$ parity is violated due to mixing, and intrinsic wavefunction of $\omega(782)$ meson would change for enlarged $m_{u} \neq m_{d}$ mass difference. Decays $\omega(782) \rightarrow \pi^{+} \pi^{-}$would then become enhanced due to larger $\left(\tilde{\varepsilon} \cdot \tilde{\rho}_{770}\right)$ admixture in $\omega(782)$ meson state.

Similar considerations can be applied to the case of $K_{L}^{0}$ and $K_{S}^{0}$ mesons. Transitions $(d \bar{s} \rightarrow s \bar{d})$ and $(\bar{d} s \rightarrow \bar{s} d)$ between $K^{0}$ and $\bar{K}^{0}$ flavor states are allowed by weak interactions (box diagrams). Wavefunctions of neutral $K_{L}^{0}, K_{S}^{0}$ mesons would be directly affected by $\left(m_{s}-m_{\bar{s}}\right) \neq 0$ mass difference [7], or due to different effective masses of $K^{0}$ and $\bar{K}^{0}$ states in a dense (baryonic) medium.

In the following sections we discuss a specific quantum superposition phenomenon, occurring in the external field, which seems to allow for the enhancement of CP violating $\eta(547) \rightarrow \pi^{+} \pi^{-}$ decays to take place in magnetic fields of sufficient strength.

\section{Superposition of $\mathrm{C}$ parity eigenstates in Positronium}

The influence of external magnetic field on Positronium [8] properties was anticipated [9], and clearly observed [10] as quenching of $\left(s_{z}=0\right)$ ortho-Positronium decays $\left(e^{+} e^{-}\right) \rightarrow \gamma \gamma \gamma$ in field $B \approx 1 \mathrm{~T}$. The phenomenon arises as a consequence of the interaction of $e^{+}, e^{-}$magnetic moments $\left(\left|\mu_{e}\right|=5.79 \cdot 10^{-11} \mathrm{MeV} / \mathrm{T}\right)$ with external field, which forces $\tilde{\Psi}_{o}(J=0)$ and $\tilde{\Psi}_{p}\left(J=1, s_{z}=0\right)$ states of bound $\left(e^{+} e^{-}\right)$pair to become superpositions of $\mathrm{C}$ parity eigenstates in magnetic field [9]:

$$
\tilde{\Psi}_{o}^{[B]}=\cos (\alpha) \Psi_{o}+\sin (\alpha) \Psi_{p} \quad, \quad \tilde{\Psi}_{p}^{[B]}=\cos (\alpha) \Psi_{p}-\sin (\alpha) \Psi_{o} .
$$

Here $\Psi_{o}=(\uparrow \downarrow+\downarrow \uparrow) / \sqrt{2}$ is ortho-Positronium state with $\left(J=1, s_{z}=0\right)$ quantum numbers and $\Psi_{p}=(\uparrow \downarrow-\downarrow \uparrow) / \sqrt{2}$ is para-Positronium $(J=0)$ state (both in zero field). Mixing parameter $\sin (\alpha)=y / \sqrt{1+y^{2}}$ depends on magnetic field as $y=x /\left(1+\sqrt{1+x^{2}}\right)$, where $x=4 \mu_{e} B / \Delta E_{h f}$ (hyperfine splitting of Positronium is $\Delta E_{h f}=8.4 \cdot 10^{-10} \mathrm{MeV}$ [8]). C-parity even admixture $\Psi_{p}$ in wave function $\tilde{\Psi}_{o}^{[B]}$ of $\left(J=0, s_{z}=0\right)$ ortho-Positronium allows for decays $\tilde{\Psi}_{o}^{[B]} \rightarrow \gamma \gamma$ to occur [9].

We observe, that $\mathrm{C}$ parity restriction in $(J=1)$ Positronium decays [11] can be effectively bypassed due to quantum superposition (mixing) effect occurring in the external field. Decays $\tilde{\Psi}_{o}^{[B]} \rightarrow \gamma \gamma$ replace the original $(3 \gamma)$ decay channel easily (one has $\tilde{\Gamma}_{o}=\left(\Gamma_{o}+y^{2} \Gamma_{p}\right) /\left(1+y^{2}\right)$ for $\tilde{\Psi}_{o}^{[B]}$ decay width [8]), which can reduce the lifetime of ortho-state $\tilde{\Psi}_{o}^{[B]}\left(s_{z}=0\right)$ up to $\approx 500$ times.

\section{Quarkonium in magnetic field, $\eta_{c}$ and $\eta(547)$ mesons}

A superposition of neutral kaon states with different $\mathrm{C}$ parity has been discussed already by Gell-Mann and Pais [12]. Similarly to light quarks $\left(\mu_{u}=1.85 \mu_{N}, \mu_{d}=-0.97 \mu_{N}, \mu_{s}=-0.61 \mu_{N}\right)$ in hyperons, one should expect heavy constituent quarks in Quarkonium mesons to have their magnetic moments $\mu_{c} \approx 0.4 \mu_{N}$ and $\mu_{b} \approx-0.07 \mu_{N}$ (using $\mu_{q}=\hbar Q_{q} / 2 m_{q} c$ ). The interaction of heavy 
quark magnetic moments with static magnetic field of sufficient strength $B \approx 10^{14} \mathrm{~T}$, shoud [13] result in a quantum superposition of $\eta_{c}(2980)$ meson (or $\eta_{b}(9391)$ state [1]) with corresponding $\left(s_{z}=0\right)$ substate of $\mathbf{J} / \Psi_{c \bar{c}}$ (or $\Upsilon_{b \bar{b}}(1 s)$ mesons). Rewriting equation (3.1) for Quarkonium, one has

$$
\tilde{\Psi}_{c \bar{c}}^{[B]}=\cos (\alpha) J / \Psi_{c \bar{c}}+\sin (\alpha) \eta_{c \bar{c}} \quad, \quad \tilde{\eta}_{c}^{[B]}=\cos (\alpha) \eta_{c \bar{c}}-\sin (\alpha) J / \Psi_{c \bar{c}}
$$

for eigenstates $(\uparrow \downarrow+\downarrow \uparrow) / \sqrt{2}=\mathrm{J} / \Psi_{c \bar{c}}\left(J=1, s_{z}=0\right)$ and $(\uparrow \downarrow-\downarrow \uparrow) / \sqrt{2}=\eta_{c \bar{c}}(J=0)$, and similar equation can be written for $(b \bar{b})$ mesons. Mixing parameter is $\sin (\alpha)=y / \sqrt{1+y^{2}}$ again, with $y=x /\left(1+\sqrt{1+x^{2}}\right)$ and $x=4 B \mu_{c, b} / \Delta E_{h f}^{q \bar{q}}$ [13], where hyperfine splitting is $\Delta E_{h f}^{c \bar{c}}=116 \mathrm{MeV}$ for Charmonium, and $\Delta E_{h f}^{b \bar{b}} \approx 69 \mathrm{MeV}$ for Bottomium [1]. Admixture of C parity-even $\eta_{c \bar{c}}$ state in $\tilde{\Psi}_{c \bar{c}}^{[B]}$ vector meson $\left(J=1, s_{z}=0\right)$ substate will allow the originally pure C parity-odd mesons $J / \Psi(3097)$ to decay (in magnetic field) into 2-gluon $(\mathrm{gg})$ states having $\mathrm{C}=+1$ parity [14]. This may reduce the lifetime and $\left(l^{+} l^{-}\right)$branching ratio of Quarkonium mesons $\Psi(n s)$ and $\Upsilon(n s)$ considerably, in static magnetic fields of sufficient strength $B \approx 10^{14}-10^{15} \mathrm{~T}$ [13].

At the same time, production of vector Charmonium state $\tilde{\Psi}_{c \bar{c}}^{[B]}$ in external field can become enhanced, because channel $g g \rightarrow \tilde{\Psi}_{c \bar{c}}^{[B]}\left(J=1, s_{z}=0\right)$ becomes possible in the magnetic field. Large $R_{A A} \approx 7$ enhancement of $J / \Psi$ meson production at $p_{t}<300 \mathrm{MeV}$, observed in peripheral $\mathrm{Pb}+\mathrm{Pb}$ interactions at LHC [15], can be related to Quarkonium state superpositions [13] discussed here.

The admixture of ortho-state $\Psi_{c \bar{c}}$ in $(J=0)$ eigenstate $\tilde{\eta}_{c}^{[B]}$ may have another interesting consequence: due to G-parity violating $J / \Psi(3097) \rightarrow \pi^{+} \pi^{-}$decay $[1]\left(\mathrm{BR} \approx 1.5 \cdot 10^{-4}\right)$, state $\tilde{\eta}_{c \bar{c}}^{[B]}$ given by Equation (4.1) may have $\tilde{\eta}_{c \bar{c}}^{[B]} \rightarrow \pi^{+} \pi^{-}$decay probability slightly enhanced. Decays $\eta^{\prime}(958) \rightarrow \pi^{+} \pi^{-}$and $\eta(547) \rightarrow \pi^{+} \pi^{-}$are strongly suppressed [16] by CP symmetry, and the same should apply to $\eta_{c}(2981)$ meson. We suggest the enhancement of $\eta_{c}(2981)$ and $\eta(547) \rightarrow \pi^{+} \pi^{-}$ decays (see Fig. 2) may occur in magnetic fields, due to the superposition effect discussed here.

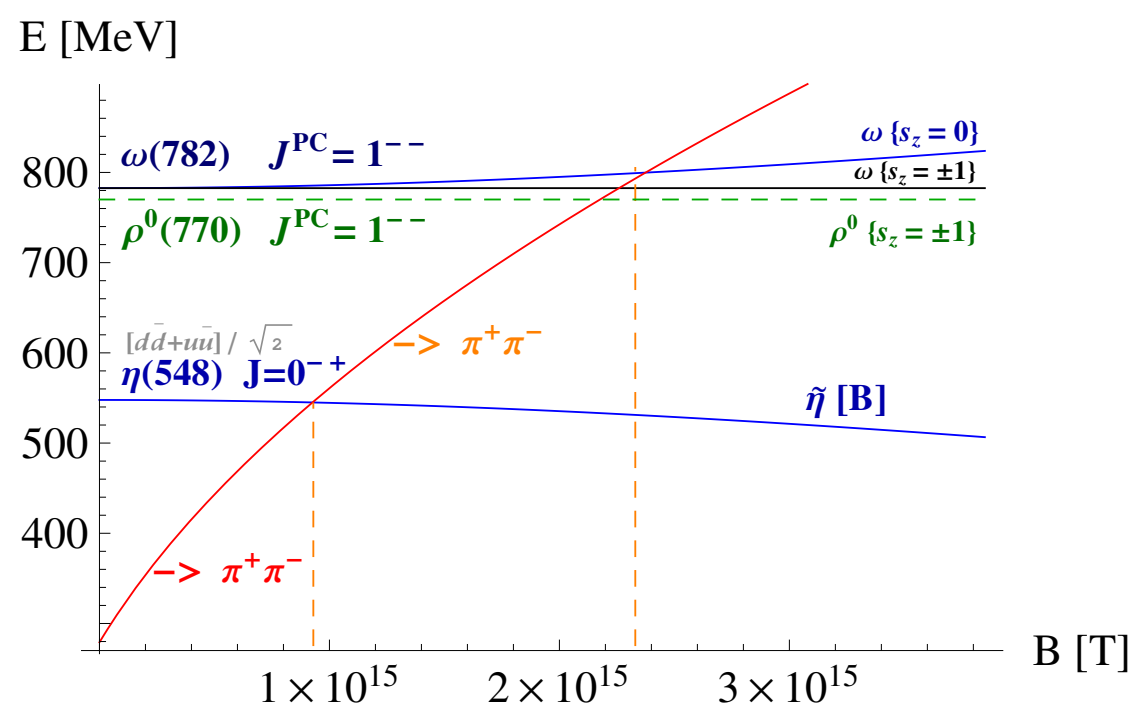

Figure 2: Energy of $\omega(782)$ and $\eta(547)$ mesons in static magnetic field in a comparison with the lowest $(n=0)$ Landau energy of decay products $\pi^{+} \pi^{-}$. Superposition of $\eta \leftrightarrow \omega_{s_{z}=0}$ mesons enhances $\eta \rightarrow \pi^{+} \pi^{-}$ decay due to $\omega$ state admixture in $\tilde{\eta}[B]$ meson. CP - violating decays $\eta \rightarrow \pi^{+} \pi^{-}$are possible for $B<10^{15} \mathrm{~T}$ only. Decay $\omega \rightarrow \pi^{+} \pi^{-}$is energetically closed at $B>2.3 \cdot 10^{15} \mathrm{~T}$, and the same applies to $\rho^{0} \rightarrow \pi^{+} \pi^{-}$[17]. 
Possibility of $\eta(547) \rightarrow \pi^{+} \pi^{-}$decay enhancement was already discussed [18], assuming $\eta(547)$ meson gets into superposition with $\left(s_{z}=0\right)$ substate of $\omega(782)$ in the magnetic field: $\tilde{\eta}[B] \approx(\eta+\varepsilon[B] \cdot \omega)$. The enhancement was estimated to be $\mathrm{BR}=F \approx 4.6 \cdot 10^{-28} B^{2} R_{\pi \pi}^{\omega}$, where $F=\Gamma_{\pi \pi}^{\tilde{\eta}}[B] / \Gamma_{\text {tot }}^{\eta}$ was branching ratio (BR) for $\tilde{\eta} \rightarrow \pi^{+} \pi^{-}$decays (using $R_{\pi \pi}^{\omega}=1.5 \%=0.015$ [1]).

The estimate in [18] was obtained assuming the rate of $\omega \rightarrow \pi^{+} \pi^{-}$(G parity violating) decays is unchanged in the magnetic field ${ }^{1}$. One can consider such estimate [18] as a lower bound for the studied effect. If we assume $\eta(547)$ state gets mixed with $\rho^{0}(770)$ meson in magnetic fields, the enhancement factor becomes larger, due to $\Gamma_{\rho^{0}}=150 \mathrm{MeV}$ decay width of $\rho^{0} \rightarrow \pi^{+} \pi^{-}$channel. One can obtain upper bounds for the studied effect, using formula derived for para-Positronium [8]

$$
\tilde{\Gamma}_{p}=\frac{\Gamma_{p}+y^{2} \Gamma_{o}}{\left(1+y^{2}\right)} \longrightarrow \quad \tilde{\Gamma}_{\tilde{\eta}}=\frac{\Gamma_{\eta}+y^{2} \Gamma_{\rho^{0}}}{\left(1+y^{2}\right)} \simeq \Gamma_{\eta}+\frac{x^{2}}{4} \Gamma_{\rho^{0}}
$$

This gives $\tilde{\Gamma}_{\tilde{\eta}}[B]=\left(1.3+1.2 \cdot 10^{-26} B^{2}\right)$ in $\mathrm{keV}$, while $\mathrm{CP}$ violation in Standard Model restricts $\eta(547) \rightarrow \pi^{+} \pi^{-} \leq 2 \cdot 10^{-27}[16]$. Assuming (roughly) magnetic moment $\tilde{\mu}_{q} \approx \mu_{N}$ for the effective "constituent quark" in considered ${ }^{2}$ meson states $(u \bar{u} \pm d \bar{d}) / \sqrt{2}$, one obtains the upper estimate for $\mathrm{CP}$ violating branching ratio $\mathrm{BR}=\Gamma_{\pi \pi}^{\tilde{\eta}}[B] / \Gamma_{\text {tot }}^{\eta} \simeq 9 \cdot 10^{-27} B^{2}$, due to $\eta(547)-\rho^{0}(770)$ mixing. The limit $\mathrm{BR} \leq 2 \cdot 10^{-27}[16]$ would then be exceeded by factor $\times 450$ in magnetic field $B \approx 10 \mathrm{~T}$.

Although magnetic fields $\approx 10 \mathrm{~T}$ are experimentally available, the amount of $\eta(547)$ meson decays required to test the predicted behavior seems to be beyond reasonable bounds. One can suggest to use large magnetic fields present in the vicinity of specific $\eta-\mathrm{Nb}$ mesic nuclei [18] to observe the effects discussed here, but we shall turn now our attention to other questions regarding the enhancement of $\mathrm{CP}$ violation in the early Universe.

\section{Discussion}

In section 2.1 we have considered $G$ parity violation due to mixing of pure eigenstates $\tilde{\omega}_{782}$ and $\tilde{\rho}_{770}^{0}$, which occurs as a consequence of light quarks mass difference $\left(m_{u} \neq m_{d}\right)$ and electromagnetc effects [6]. One may ask whether sufficiently strong magnetic field can influence $\rho^{0}-\omega$ mixing?

Due to different charges of quarks $\left(Q_{u}=2 Q_{\bar{d}}\right)$, the interaction of $u$ and $d$ quarks with magnetic field (rising the Landau level energy) will be different, and thus our answer is positive. Moreover, states $(q \bar{q})_{J, s_{z}}^{[B]}$ in the magnetic field become quantum superpositions: $(q \bar{q})_{1,0}^{[B]} \approx(q \bar{q})_{1,0}+\tilde{\varepsilon}_{q}(q \bar{q})_{0,0}$ (similarly to ortho-Charmonium), and transition process $(u \bar{u})_{1,0}^{[B]} \rightarrow\{2 g\} \rightarrow(d \bar{d})_{1,0}^{[B]}$ should become allowed in the magnetic field (proportionally to $\tilde{\varepsilon}^{4}$ ), what can affect degree of $\rho^{0}-\omega$ mixing.

Can sufficiently strong magnetic fields influence also weak $\left(K^{0} \pm \bar{K}^{0}\right)$ mixing phenomena? Can degree of CP violation in CKM matrix be affected or induced by some kind of external field? Answers to these questions are clearly [19] beyond the scope of this contribution. We just make a short speculative comment here: Penguin and box diagrams [20] used to calculate CP violating processes in Standard Model regularly contain closed loops of $\left(W^{+}, W^{-}\right.$, quark and antiquark) particle propagators. In the external field, particles possessing electric charge $Q_{e}$ (or weak isospin

\footnotetext{
${ }^{1}$ Strangeness $(s \bar{s})$ content in $\eta(547)$ was neglected, and effective magnetic moment $\mu_{q}=\mu_{u}+\mu_{d} \approx 0.9 \mu_{N}$ was assumed for a "constituent quark" in the considered $\omega=(u \bar{u}+d \bar{d}) / \sqrt{2}$ meson and $\tilde{\eta} \approx(u \bar{u}+d \bar{d}) / \sqrt{2}$ state (see Fig. 2).

${ }^{2}$ In extremal magnetic fields, isospin structure of light mesons gets modified due to different charges of $u, d$ quarks.
} 
charge $Q_{w}$ ) acquire quantum phase $\varphi=Q_{w, e} \int \vec{A}_{w, e} d \vec{s} / \hbar$, where $\vec{A}_{w, e}$ stands for the vector potential of electromagnetic or weak fields.

Closed loops of combined particle propagators may allow for the acquired quantum phase $\Delta \varphi$ to be untransformable, permitting the specific type of phenomena (possibly related to $\mathrm{CP}$ violation) to occur. Magnitude of such electroweak fields (potentials) in the expanding Universe could be time dependent, giving larger intensity for CP - type violation effects in the early state of Universe after Big Bang, when the asymmetry between matter and antimatter was generated.

We finish our speculations by mentioning the possibility that cosmological electroweak fields may naturally appear also in the later stages of the rotating Universe evolution, due to known behavior of quantum condensates, allowing for London-type [21] magnetic fields $\vec{B}_{L}=2 m_{B} \vec{\omega} / q$ to permeate the volume of the rotating condensate.

\section{Acknowledgements}

This work and active participation at EPS-HEP 2015 conference in Vienna were financially supported by the Slovak Grant Agency (VEGA) within project N. 2/0197/14.

\section{References}

[1] J. Beringer et al. (Particle Data Group), Physical Review D86, 1 (2012).

[2] J. H. Christenson et al., Phys. Rev. Lett. 13, 138 (1964).

[3] A. Alavi-Harati et al. (KTeV Collab.), Phys. Rev. Lett. 83, 22 (1999).

[4] A. Yaounac, L. Oliver, O. Pene, J.-C. Raynal, Phys. Rev. D8, 2223 (1973).

[5] Ch. Vaissière, Il Nuovo Cimento 41A, 419 (1977).

[6] S.L. Glashow, Phys. Rev. Lett. 7, 469 (1961).

[7] M.J. Fromerth and J. Rafelski, Acta Physica Polonica B34, 4151 (2002).

[8] A.Rich, Rev. Mod. Phys. 53, 127 (1981).

[9] O.Halpern, Physical Review 94, 904 (1954).

[10] V.W.Hughes, S.Marder and C.S.Wu, Physical Review 98, 1840 (1955).

[11] P.A. Vetter, Modern Physics Letters A19, 871 (2004).

[12] M. Gell-Mann and A. Pais, Physical Review 97, 1387 (1955).

[13] P. Filip, Proceedings of Science, PoS (CPOD 2013) 035.

[14] V.A. Novikov et al., Physics Reports 41C, 1 (1978).

[15] J. Adam et al. (ALICE Collab.), preprint CERN-PH-EP-2015-268; [arXiv:1509.08802].

[16] C. Jarlskog and E. Shabalin, Phys. Rev. D52, 248 (1995).

[17] M.N. Chernodub, Physical Review D82, 085011 (2011).

[18] P. Filip, EPJ Web of Conferences 81, 05013 (2014).

[19] H. Georgi and A. Pais, Physical Review D10, 1246 (1974).

[20] G.C. Branco, L. Lavoura and J.P. Silva, CP-violation, (Oxford University Press, Oxford, 1999).

[21] R.M. Brady, Journal of Low Temperature Physics 49, 1 (1982). 